\title{
Dynamic protein interaction modules in human hepatocellular carcinoma progression
}

\author{
Hui Yu', Chen-Ching Lin', Yuan-Yuan Li ${ }^{2}$, Zhongming Zhao ${ }^{1,3,4,5^{*}}$ \\ From The International Conference on Intelligent Biology and Medicine (ICIBM 2013) \\ Nashville, TN, USA. 11-13 August 2013
}

\begin{abstract}
Background: Gene expression profiles have been frequently integrated with the human protein interactome to uncover functional modules under specific conditions like disease state. Beyond traditional differential expression analysis, differential co-expression analysis has emerged as a robust approach to reveal condition-specific network modules, with successful applications in a few human disease studies. Hepatocellular carcinoma (HCC), which is often interrelated with the Hepatitis C virus, typically develops through multiple stages. A comprehensive investigation of HCC progression-specific differential co-expression modules may advance our understanding of HCC's pathophysiological mechanisms.

Results: Compared with differentially expressed genes, differentially co-expressed genes were found more likely enriched with Hepatitis C virus binding proteins and cancer-mutated genes, and they were clustered more densely in the human reference protein interaction network. These observations indicated that a differential co-expression approach could outperform the standard differential expression network analysis in searching for disease-related modules. We then proposed a differential co-expression network approach to uncover network modules involved in HCC development. Specifically, we discovered subnetworks that enriched differentially co-expressed gene pairs in each HCC transition stage, and further resolved modules with coherent co-expression change patterns over all HCC developmental stages. Our identified network modules were enriched with HCC-related genes and implicated in cancer-related biological functions. In particular, APC and YWHAZ were highlighted as two most remarkable genes in the network modules, and their dynamic interaction partnership was resolved in HCC development.

Conclusions: We demonstrated that integration of differential co-expression with the protein interactome could outperform the traditional differential expression approach in discovering network modules of human diseases. In our application of this approach to HCC's gene expression data, we successfully identified subnetworks with marked differential co-expression in individual HCC stage transitions and network modules with coherent coexpression change patterns over all HCC developmental stages. Our results shed light on subtle HCC mechanisms, including temporal activation and dismissal of pivotal functions and dynamic interaction partnerships of key genes.
\end{abstract}

\section{Background}

With great improvement in both the quantity and quality of human protein-protein interaction data, a comprehensive human protein interaction network was created and serves as the backbone of many human disease studies [1-4]. However, the reference protein interaction

\footnotetext{
* Correspondence: zhongming.zhao@vanderbilt.edu

'Department of Biomedical Informatics, Vanderbilt University School of Medicine, Nashville, TN 37203, USA

Full list of author information is available at the end of the article
}

network masks the in vivo spatial and temporal contexts and unrealistically integrates all in vitro molecular interactions together. Therefore, it is imperative to identify condition-specific protein interaction network modules that have more spatiotemporal biological relevance to disease studies. Recently, in response to the call for dynamic interactomes $[5,6]$, many efforts have been made to extract active subnetworks by integrating stagewise or time series gene expression data into protein interaction network [7-20]. 
As the most intuitive and straightforward expression feature, differential expression statistics are often overlaid onto protein interaction network, and subnetworks enriched for differential expression genes are fetched [7-11]. However, since protein interaction network is essentially a model of relations among biological molecules, a more precise characterization of dynamic protein interaction network would result from addressing the relation changes than the entity changes. The differential co-expression analysis, which investigates the changes of expression correlations between genes, has arisen as a promising alternative to traditional differential expression analysis [21]. While differential co-expression is related to co-expression, the gene-gene co-expression in a comparative condition should not be a prerequisite [22,23]. In relation to protein interaction network studies, however, co-expression analyses were more frequently performed than differential co-expression analyses [12-16]. Recently, the use of differential co-expression analyses to uncover dynamic protein interaction network modules specific to human diseases has begun [17-20]. These studies have made important discoveries in heart failure [18], glioma prognosis [17], HIV infection [19], and other diseases. However, some of these studies were predisposed to co-expression analysis, which may have limited the potential of their approaches.

Hepatocellular carcinoma (HCC) is the most common type of liver cancer, and it is often interrelated with the Hepatitis $C$ virus (HCV). A typical HCC progression may go through the following successive stages: Normal $(\mathrm{N})$, Cirrhosis (C), Dysplasia (D), Early HCC (E), and Advanced HCC (A). As a gradually-developed carcinoma with marked pre-neoplastic stages and neoplastic stages, HCC calls for a better understanding at the genomic level of the origin and transitions of its carcinogenesis. A welldesigned, multi-stage HCC expression dataset has been analyzed by different groups from the perspective of differential expression with [24] or without network context [25], but the dataset has not been explored for differential co-expression yet. Like the successful attempt in a multistate human colorectal cancer study [20], a differential co-expression analysis of protein interaction network modules may advance our understanding of HCC's pathophysiological mechanisms.

In this study, we first comparatively evaluated Differentially Expressed Genes (DEGs) and Differentially Co-expressed gene Pairs (DCPs) for their qualification for subnetwork seeds, and as a result proved the improved validity of searching for protein interaction subnetworks from seeds of DCPs than DEGs. We then proposed a differential co-expression network approach to uncover gene modules involved in HCC development. Specifically, we identified subnetworks that enriched DCPs in each HCC transition stage, and further resolved modules with coherent co-expression change patterns over all HCC developmental stages (Figure 1). Our identified network modules were found to be enriched with HCC-related genes and implicated in cancer-related biological functions. The results shed light on subtle HCC mechanisms, including temporal activation and dismissal of pivotal functions and dynamic interaction partnerships of key genes.

\section{Results}

Differential co-expression protein interactions as seeds in subnetwork searches

The stage-wise Pearson correlation coefficient $(r)$ values of gene expression profiles were calculated for all possible pairs formed by the genes in the reference protein interaction network. For each HCC stage, we compared all pairs' Pearson correlation coefficients (absolute values) with those of the protein interaction pairs' subset (absolute values) using the two-sample KolmogorovSmirnov test. We found that at all stages the protein interaction pairs' absolute Pearson correlation coefficients were larger than the total control at a significant level ( $p<0.001$ for N, C, and D) or marginally significant level $(p<0.1$, for $\mathrm{E}$ and $\mathrm{A})$.

Then, we derived the differential correlation values $(d C)$ for each protein interaction pair and each stage transition. In this manner, each protein interaction pair was described with a vector of four $d C$ values. A sizeable portion of distant protein interaction pairs characterized with noticeably larger $d C$ values were discovered in an outlier analysis of the $d C$ data matrix (Additional file 1).

Most approaches to extracting protein interaction subnetworks, including our previous one [24], were seeded from a set of DEGs. In this work, we intended to use DCPs as alternative seeds; therefore, we initially set out to investigate if DCPs were a better option for seeds. Successively inclusive sets of top-DEGs (based on absolute log fold changes of mean expression) or top-DCPs (based on absolute $d C$ values) were chosen at three increasing levels: $0.1 \%, 0.5 \%$, or $1 \%$. For comparability, we derived corresponding sets of "Differentially Coexpressed Genes" (DCGs) as the genes involved in DCPs and compared the derived DCGs with the DEGs at the same levels.

First, an ideal seed gene set should be related to the studied subject, which in our case was the HCV-induced HCC progression. For this criterion, we evaluated topDEGs and top-DCGs in terms of their enrichment of $\mathrm{HCV}$-protein-binding $(\mathrm{HCB})$ proteins, cancer-mutated genes from Cancer Gene Consensus (CGC), or HCCresponsive genes (HCR). As clearly shown in Table 1 HCV-binding proteins were more enriched in top-DCGs than in top-DEGs: except for one case, all top-DCG sets 


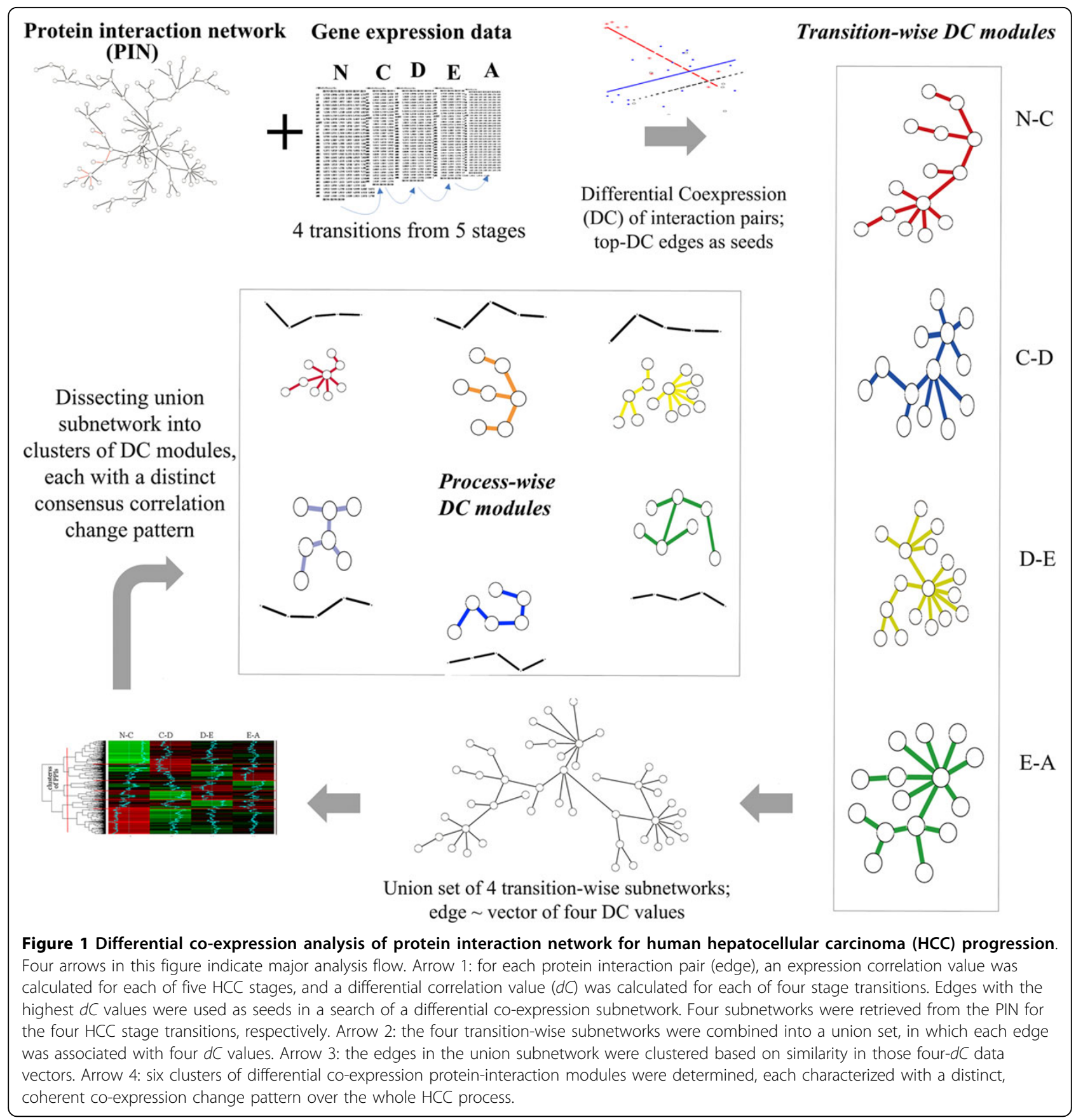

were significantly enriched with HCV-binding proteins, whereas none of top-DEG sets were enriched with HCVbinding proteins. In terms of HCC-responsive genes and cancer-mutated genes, advantages were still existent using DCGs compared to DEGs (Additional file 1).

Additionally, as many subnetwork-searching algorithms (e.g. Steiner minimum tree [26]) implicitly assume, a set of seed genes should ideally cluster densely in the whole network so that they can be connected into a subnetwork via a limited number of mediators. It therefore follows that a set of seed genes should have an average pairwise distance shorter than that between random pairs. As a baseline, the shortest paths in the whole protein interaction network were $3.81 \pm 0.86$ (any disconnected protein pairs that were unable to be connected via any path were excluded in this calculation). We found that the shortest paths among DCG seeds were generally one-step shorter than random pairs, while those among DEG seeds were generally very close to random pairs (Table 2). In fact, the advantage of 
Table 1 Proportion of Hepatitis $C$ virus-binding proteins in top ranked gene seeds

\begin{tabular}{llllll}
\hline Top level $^{\mathbf{a}}$ & Gene set & & \multicolumn{3}{c}{ HCC transition } \\
\cline { 3 - 6 } & & N-C & C-D & D-E & E-A \\
\hline $0.1 \%$ & DEG & $0 / 11$ & $1 / 11$ & $0 / 11$ & $1 / 11$ \\
& DCG & $7 / 63^{*}$ & $9 / 65^{* * *}$ & $10 / 60^{* * *}$ & $4 / 63$ \\
$0.5 \%$ & DEG & $2 / 55$ & $2 / 55$ & $2 / 55$ & $4 / 55$ \\
& DCG & $25 / 290^{* * *}$ & $36 / 279^{* * *}$ & $27 / 287^{* * *}$ & $27 / 288^{* * *}$ \\
$1.0 \%$ & DEG & $6 / 110$ & $3 / 110$ & $5 / 110$ & $6 / 110$ \\
& DCG & $47 / 524^{* * *}$ & $51 / 538^{* * *}$ & $36 / 523^{* * *}$ & $41 / 559^{* * *}$ \\
\hline
\end{tabular}

In each cell, the proportion is shown as "x/y", with y for top-ranked genes and $\times$ for genes of interest. Enrichment $p$-value $<0.01(*)$ or $<0.0001(* *)$ are marked. ${ }^{a}$ Top-level is the fraction of the top-ranked genes/pairs in the total network nodes/edges. For differential expression genes (DEG), we used the node-wise fraction; for differential co-expression genes (DCGs) deriving from pairs, we used the edge-wise fraction. A same fraction of pairs involve a greater number of genes.

${ }^{\mathrm{b}}$ Transition names: Normal to Cirrhosis (N-C), Cirrhosis to Dysplasia (C-D), Dysplasia to Early HCC (D-E), and Early HCC to Advanced HCC (E-A).

DCG seeds over DEG seeds was underestimated here, as disconnected pairs were found in DEG sets but not in DCG sets (footnotes of Table 2). In conclusion, the seed DCGs were clustered more densely in protein interaction network than the seed DEGs, implying a more likely formation of compact, expression-coherent subnetworks when using seed DCPs than seed DEGs.

Therefore, the relative advantage of DCPs as seeds in protein interaction subnetwork searches was demonstrated against traditional DEGs, which justified our subsequent edge-wise subnetwork searches from these top-ranked seed DCPs.

\section{Differential co-expression subnetwork in each HCC stage-transition}

In order to avoid oversized subnetworks, we used the top 0.1\% DCPs (65 DCPs) as seeds in the subnetwork searches (see Materials and methods). These top 65 DCPs involved $123,124,118$, and 123 seed genes in the four stage transitions, respectively (Additional file 2).

One subnetwork was retrieved for each transition between consecutive HCC stages, and the four subnetworks are termed "transition-wise" subnetworks hereafter. The properties of these transition-wise subnetworks are summarized in Table 3 and Additional file 1, and full subnetworks can be reviewed in Additional files 3 and 4 . While the overall edge-to-node ratio in protein interaction network is about $5.9(64,865$ to 10,953$)$, the same statistics in the subnetworks is about 1 , reflecting a selective recruitment of edges into the subnetworks. The $r$ values and $d C$ values associated with links in the subnetworks are more conspicuous than the background level in protein interaction network (Additional file 1), indicating an effective condensation of differential co-expression relations.

The coverage of HCC-related genes in the transitionwise subnetworks was studied to evaluate the relevance of our subnetworks in relation to HCC development. Three types of HCC-related genes, HCB, CGC, and HCR, were examined separately. Almost all four transition-wise subnetworks were enriched with these HCC-related genes (Table 3). Then, following the example of our previous work [24], we defined proteins with more than six connections as hubs and obtained a total of 25 hubs in the four subnetworks (Table 3). Among the 18 Gene Ontology (GO) [27] biological processes terms enriched within these hub genes (Additional file 1), some are evidently related to HCC pathogenesis, such as "interspecies interaction between organisms", "immune response-activating signal transduction" [28], and "platelet activation" [29]. In summary, nine hubs are targeted by HCV proteins, and five are mutated in cancer. Of the only five liver-cancer-associated genes from CGC, APC [30] appeared as a recurrent hub in multiple subnetworks. These observations suggested that our transition-wise

Table 2 Average shortest length among top ranked genes

\begin{tabular}{llllll}
\hline Top level & Gene set & \multicolumn{3}{c}{ HCC transition } \\
\cline { 3 - 6 } & & N-C & C-D & D-E & E-A \\
\hline $0.1 \%$ & DEG & $3.61 \pm 0.59$ & $3.25 \pm 0.86$ & $3.40 \pm 0.91$ & $4.25 \pm 0.86$ \\
& DCG & $2.96 \pm 0.71$ & $2.94 \pm 0.74$ & $2.72 \pm 0.75$ & $2.98 \pm 0.75$ \\
$0.5 \%$ & DEG & $3.70 \pm 0.78^{\mathbf{a}}$ & $3.62 \pm 0.85$ & $3.76 \pm 0.82$ & $3.62 \pm 0.85^{\mathbf{b}}$ \\
& DCG & $2.89 \pm 0.71$ & $2.81 \pm 0.68$ & $2.82 \pm 0.73$ & $2.89 \pm 0.70$ \\
$1.0 \%$ & DEG & $3.60 \pm 0.81^{\mathbf{c}}$ & $3.63 \pm 0.84$ & $3.67 \pm 0.81^{\mathbf{d}}$ & $3.60 \pm 0.87 \mathbf{e}$ \\
& DCG & $2.87 \pm 0.69$ & $2.85 \pm 0.76$ & $2.86 \pm 0.70$ & $2.90 \pm 0.71$ \\
\hline
\end{tabular}

There were $3.6 \%^{\mathrm{a}}, 10.7 \%^{\mathrm{b}}, 1.8 \%{ }^{\mathrm{c}}$, or $5.4 \%^{\mathrm{d}, \mathrm{e}}$ disconnected DEG pairs and they were excluded from the average shortest length calculation. 
Table 3 Transition-wise differential co-expression protein interaction subnetworks

\begin{tabular}{|c|c|c|c|c|c|c|}
\hline \multirow[t]{2}{*}{ Transition } & \multirow[t]{2}{*}{$\begin{array}{l}\# \\
\text { nodes }\end{array}$} & \multirow[t]{2}{*}{$\begin{array}{l}\# \\
\text { edges }\end{array}$} & \multicolumn{3}{|c|}{$\begin{array}{l}\text { HCC-related } \\
\text { genes }^{\text {a }}\end{array}$} & \multirow[t]{2}{*}{ Hub genes ${ }^{b}$} \\
\hline & & & $\overline{\mathrm{HCB}}$ & CGC & HCR & \\
\hline $\mathrm{N}-\mathrm{C}$ & 307 & 310 & $28^{* * *}$ & $29 * * *$ & $154^{*}$ & $\begin{array}{l}\text { ARRB1, APC, CDKN2A, CSNK2A1, CSNK2A2, EP300, ESR1, GRB2, HCK, MCC, PIK3R1, PPP1CC, SRRM2, } \\
\text { SUMO2, TSC22D1, YWHAG, YWHAZ }\end{array}$ \\
\hline C-D & 102 & 103 & $13^{* * *}$ & $10^{*}$ & 44 & APC, GFI1B, NEDD4, UBE2D3 \\
\hline D-E & 104 & 103 & $10^{*}$ & $13^{* * *}$ & $58^{*}$ & IKBKE, PIK3R1, YWHAZ \\
\hline E-A & 103 & 102 & $9 *$ & $11^{*}$ & $60^{*}$ & EGFR, FYN, IKBKG, PRMT1,YWHAG \\
\hline
\end{tabular}

${ }^{a}$ See Materials and methods for explanation of the three HCC-related gene sets. Significance levels of HCC-related gene enrichment are labeled by * $\left.p<0.01\right)$ and ${ }^{* * *}(p<0.0001)$.

${ }^{\mathrm{b}}$ Hubs are genes with six or more connected neighbors. Hepatitis $\mathrm{C}$ virus -binding genes (italicized) and cancer-mutated genes (bold) are marked.

subnetworks are highly relevant to the development of HCC.

Then, we investigated the overlapping genes and edges between transition-wise subnetworks. There was a moderate overlap in subnetwork nodes (as high as $28.4 \%$ ) but a minor overlap in subnetwork edges (as high as 13.6\%). Altogether, we observed 16 differentially co-expressed protein interaction pairs recurrent in multiple subnetworks, two of which involved $\mathrm{N}-\mathrm{C}$ and $\mathrm{E}-\mathrm{A}$ transitions, and the other 14 of which involved consecutive N-C and C-D transitions (Additional file 1). For all $14 \mathrm{~N}-\mathrm{C}-\mathrm{D}$ continuous-changing protein interaction pairs, the expression correlation values reached a significantly high level in cirrhosis but not in normal or dysplasia stages (FDR threshold of 0.25 , equivalent to $|r|>0.76$ in cirrhosis). Remarkably, seven of these 14 protein interaction pairs were connected into a APC-centered module (Figure 2), in which four proteins, APC, CYTH2, ARRB2, and CTNNA1, were involved in the "Signaling events mediated by Hepatocyte Growth Factor Receptor (c-Met)" [31]. Aside from the important core protein APC, another protein CTNNA1 may be worth special attention as well, as it takes part in the E-cadherin/catenin complex whose abrogation was implicated in the carcinogenesis of several malignancies [32]. The aggregation of differential co-expression relations around the HCC-mutated gene $A P C$ and the involvement of quite a few HCC-related genes suggest that the APCcentered protein interaction module (Figure 2) may encode pivotal HCC-pathogenesis mechanisms for which further investigation is warranted.

\section{Differential co-expression modules in HCC progression process}

While the transition-wise analysis reveals differential coexpression subnetworks that are remarkable in single stage transitions, a process-wise analysis may catalogue protein interaction network modules based on their co-expression change patterns over all HCC development stages. Therefore, we obtained the union of the four transition-wise subnetworks and dissected them into clusters of modules

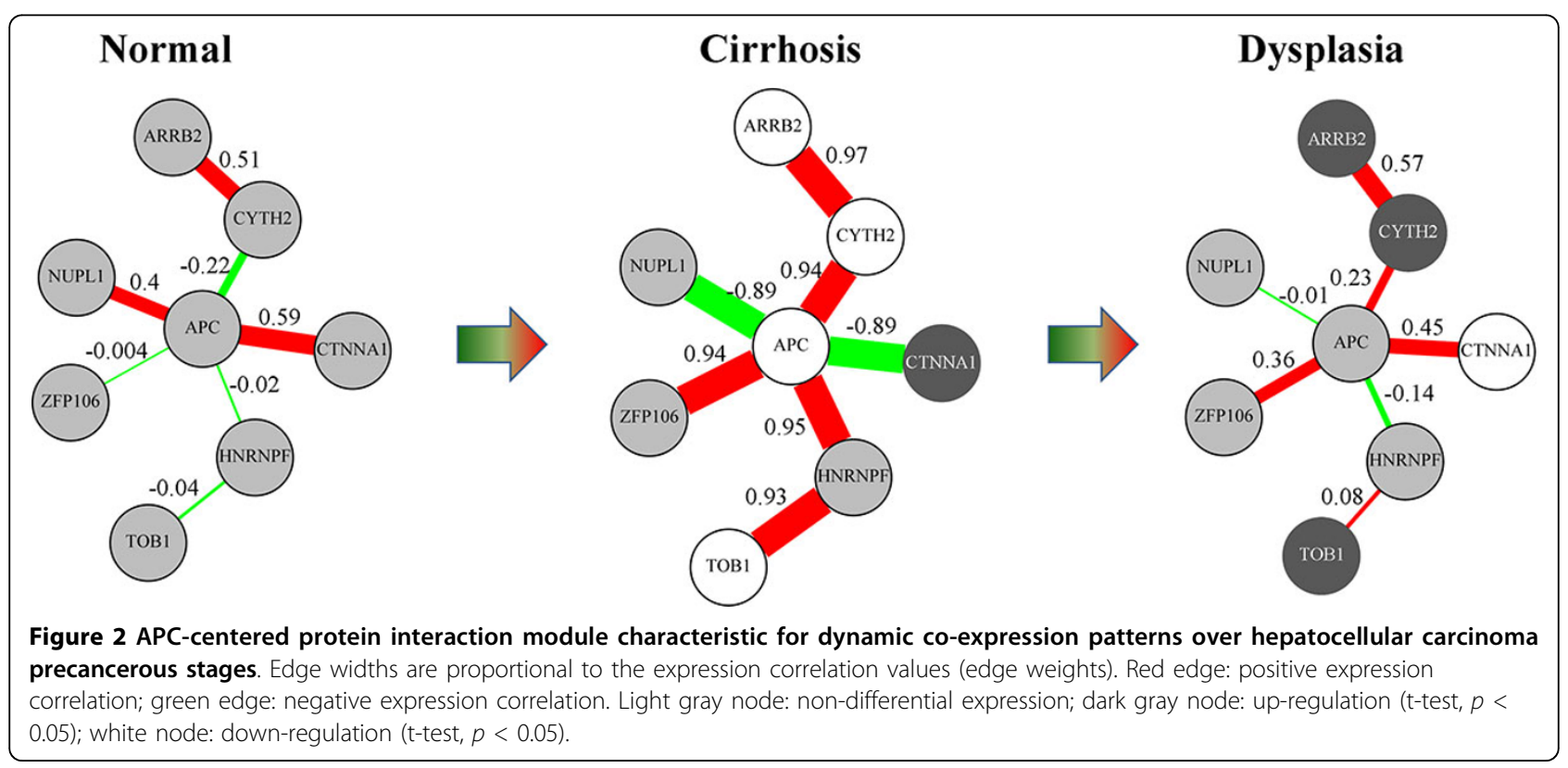




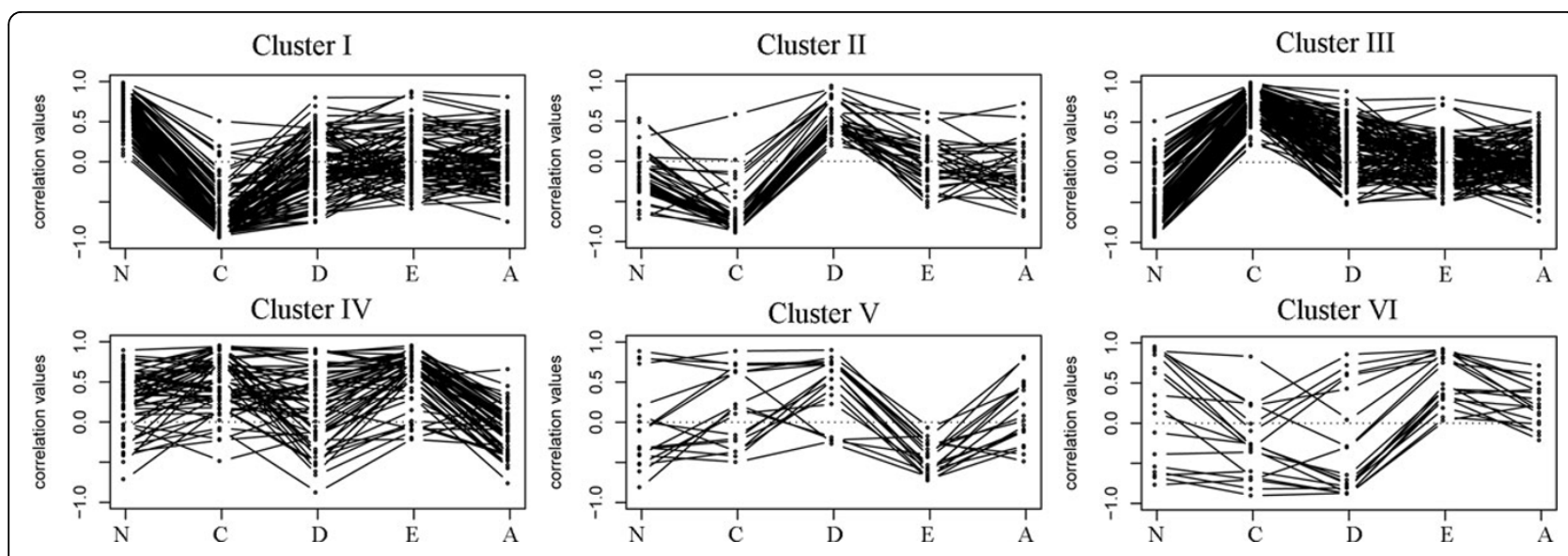

Figure 3 Expression correlation change patterns of six clusters of differential co-expression protein interaction modules. Abscissa includes five Hepatocellular carcinoma (HCC) stages: Normal (N), Cirrhosis (C), Dysplasia (D), E (Early HCC), and A (Advanced HCC).

by clustering the vectors of $d C$ values associated with each protein interaction pair (Euclidean distance measure; complete linkage clustering). This approach resulted in six clusters of protein interaction pairs with mutually distinct correlation change patterns (Figure 3), where each cluster was comprised of multiple disconnected network modules (Table 4 and Additional files 5 and 6). Further investigation revealed the dynamic correlation change patterns of each cluster (Figure 3). Clusters I, II, and III were more dynamic in early, precancerous phases (N-C-D), while clusters IV, V, and VI were more dynamic in later, cancerous phases (D-E-A).

A functional enrichment analysis was performed to uncover the biological themes of each cluster (see Materials and methods), and the results are summarized in Table 5. In coherence with $\mathrm{HCV}$-associated $\mathrm{HCC}$ progression, some relevant biological processes were discovered, such as "viral reproduction" (cluster I), "interspecies interaction between organisms" (cluster III and cluster IV), and "wound healing" (cluster V) [33].

Among the early-active clusters, cluster I and cluster III are representatives of two opposite trends: when the disease progresses from the normal stage through cirrhosis to dysplasia, Pearson correlation coefficient values in cluster III go upward and then downward, while those in cluster I show a trend that is exactly reversed. Interestingly, some enriched functions of these two clusters happen to be contrary to each other (Table 5). For instance, "negative regulation of apoptotic process" is enriched in cluster I, while "negative regulation of cell proliferation" is enriched in cluster III. It seems that, at the precancerous stages of HCC, the cells are coordinating some proliferation-inhibiting genes while simultaneously dismissing some apoptosis-inhibiting genes. These functions are possibly spontaneous calibration mechanisms taking place in precancerous stages to "halt" the potential carcinogenesis. As another probable calibration action, expression coordination is enhanced in "positive regulation of apoptotic process" (cluster II) at a later precancerous stage, dysplasia.

Of the three later-activated clusters, cluster V, where protein interaction pairs undergo consistent correlation enhancements from early HCC to advanced HCC (Figure 3), is enriched with the greatest number of functional terms (Table 5). Seven genes in cluster $\mathrm{V}$ are involved in "negative regulation of apoptotic process", and their enhanced correlations in the advanced HCC samples likely indicate an ultimate breakdown of the apoptosis program. Other potentially relevant terms tagged to cluster V include "wound healing" and "MAPK cascade,"

Table 4 Six clusters of process-wise differential co-expression protein interaction modules

\begin{tabular}{|c|c|c|c|c|c|}
\hline Cluster ID & \# node & \# edge & $\begin{array}{l}\text { \# components } \\
\text { (size } \geq 5)\end{array}$ & Size of largest component & \# HCV-binding genes \\
\hline I & 96 & 87 & 10 & 13 & 6 \\
\hline$\|$ & 40 & 36 & 4 & 20 & $9^{* * *}$ \\
\hline III & 122 & 112 & 10 & 21 & $14^{* * *}$ \\
\hline IV & 60 & 56 & 4 & 19 & $8 *$ \\
\hline V & 21 & 18 & 3 & 7 & $3 *$ \\
\hline VI & 20 & 18 & 2 & 13 & 2 \\
\hline
\end{tabular}

Hepatitis $C$ virus (HCV)-binding protein enrichment significance levels: ${ }^{*}$ for $p<0.01$ and ${ }^{* * *}$ for $p<0.0001$. 


\section{Table 5 Gene Ontology (GO) Biological Processes enriched in clusters of differential co-expression protein interaction} modules

\begin{tabular}{|c|c|c|c|c|c|}
\hline Cluster & GOID & Term & \# expec-ted genes & \# genes & Adjusted $p$-value * \\
\hline I & GO:0071842 & cellular component organization at cellular level & 23.3 & 44 & 0.0002 \\
\hline । & GO:0007346 & regulation of mitotic cell cycle & 2.6 & 12 & 0.0004 \\
\hline । & GO:2000241 & regulation of reproductive process & 1.2 & 8 & 0.0006 \\
\hline । & GO:0009968 & negative regulation of signal transduction & 4.3 & 15 & 0.0006 \\
\hline । & GO:0043066 & negative regulation of apoptotic process & 4.9 & 16 & 0.0006 \\
\hline । & GO:0031577 & spindle checkpoint & 0.4 & 5 & 0.0007 \\
\hline । & GO:0032088 & negative regulation of NF-kappaB transcription factor activity & 0.4 & 5 & 0.0007 \\
\hline I & GO:0000086 & G2/M transition of mitotic cell cycle & 1.3 & 8 & 0.0007 \\
\hline । & GO:0048522 & positive regulation of cellular process & 23.2 & 41 & 0.0007 \\
\hline । & GO:0006366 & transcription from RNA polymerase II promoter & 10.2 & 24 & 0.0007 \\
\hline । & GO:0042221 & response to chemical stimulus & 20.2 & 37 & 0.0008 \\
\hline । & GO:0031623 & receptor internalization & 0.4 & 5 & 0.0008 \\
\hline । & GO:0048468 & cell development & 10.5 & 24 & 0.0008 \\
\hline । & GO:0050658 & RNA transport & 1.0 & 7 & 0.0008 \\
\hline । & GO:0016032 & viral reproduction & 3.7 & 13 & 0.0009 \\
\hline$\|$ & GO:0043065 & positive regulation of apoptotic process & 2.0 & 11 & 0.0003 \\
\hline$\|$ & GO:0006366 & transcription from RNA polymerase II promoter & 4.3 & 15 & 0.0005 \\
\hline III & GO:0016567 & protein ubiquitination & 4.5 & 17 & 0.0001 \\
\hline III & GO:0000075 & cell cycle checkpoint & 2.7 & 13 & 0.0001 \\
\hline III & GO:0042981 & regulation of apoptotic process & 12.9 & 30 & 0.0001 \\
\hline III & GO:0000165 & MAPK cascade & 4.4 & 16 & 0.0001 \\
\hline III & GO:0032268 & regulation of cellular protein metabolic process & 12.6 & 29 & 0.0001 \\
\hline III & GO:0010627 & regulation of intracellular protein kinase cascade & 5.7 & 18 & 0.0002 \\
\hline III & GO:0050863 & regulation of $\mathrm{T}$ cell activation & 2.4 & 11 & 0.0002 \\
\hline III & GO:0007346 & regulation of mitotic cell cycle & 3.3 & 13 & 0.0002 \\
\hline III & GO:0044419 & interspecies interaction between organisms & 4.4 & 15 & 0.0003 \\
\hline III & GO:0006511 & ubiquitin-dependent protein catabolic process & 4.0 & 14 & 0.0003 \\
\hline III & GO:0045892 & negative regulation of transcription, DNA-dependent & 7.7 & 20 & 0.0005 \\
\hline III & GO:0007265 & Ras protein signal transduction & 2.8 & 11 & 0.0006 \\
\hline III & GO:0007173 & epidermal growth factor receptor signaling pathway & 1.9 & 9 & 0.0007 \\
\hline III & GO:0051090 & regulation of sequence-specific DNA binding transcription factor activity & 3.3 & 12 & 0.0007 \\
\hline III & GO:0009967 & positive regulation of signal transduction & 6.7 & 18 & 0.0007 \\
\hline III & GO:0016310 & phosphorylation & 13.6 & 28 & 0.0008 \\
\hline III & GO:0045732 & positive regulation of protein catabolic process & 0.8 & 6 & 0.0008 \\
\hline III & GO:0030518 & intracellular steroid hormone receptor signaling pathway & 1.1 & 7 & 0.0008 \\
\hline III & GO:0042770 & signal transduction in response to DNA damage & 1.5 & 8 & 0.0009 \\
\hline III & GO:0080134 & regulation of response to stress & 6.9 & 18 & 0.0009 \\
\hline III & GO:0008285 & negative regulation of cell proliferation & 5.1 & 15 & 0.0009 \\
\hline $\mathrm{IV}^{*}$ & GO:0044419* & interspecies interaction between organisms & 2.1 & 11 & 0.0020 \\
\hline $\mathrm{IV}^{*}$ & GO:0000904 * & cell morphogenesis involved in differentiation & 3.3 & 12 & 0.0099 \\
\hline $\mathrm{IV}^{*}$ & GO:0007165* & signal transduction & 18.8 & 33 & 0.0099 \\
\hline V & GO:0065008 & regulation of biological quality & 4.1 & 13 & 0.0008 \\
\hline V & GO:0001775 & cell activation & 1.4 & 8 & 0.0009 \\
\hline V & GO:0018193 & peptidyl-amino acid modification & 1.0 & 7 & 0.0009 \\
\hline V & GO:0048011 & nerve growth factor receptor signaling pathway & 0.5 & 5 & 0.0009 \\
\hline V & GO:0043066 & negative regulation of apoptotic process & 1.1 & 7 & 0.0009 \\
\hline V & GO:0000165 & MAPK cascade & 0.8 & 6 & 0.0009 \\
\hline V & GO:0042060 & wound healing & 1.2 & 7 & 0.0009 \\
\hline V & GO:0045860 & positive regulation of protein kinase activity & 0.8 & 6 & 0.0009 \\
\hline V & GO:0071375 & cellular response to peptide hormone stimulus & 0.5 & 5 & 0.0009 \\
\hline
\end{tabular}

*Threshold value for adjusted $p$-value is 0.01 (Cluster IV) or 0.001 (clusters I, II, III, and V). No GO term was found to be enriched for cluster VI with either threshold value. 
which are functions frequently implicated in carcinogenesis or cancer metastasis [33,34]. Cluster IV, a group of protein interaction pairs with collapsed correlations in advanced $\mathrm{HCC}$, is enriched with "interspecies interaction between organisms."

Interestingly, 27 interfacing proteins were found interacting with partners from different dynamic co-expression clusters, which contain both highly-connected proteins and lowly connected proteins. Ten proteins (YWHAZ, TSC22D1, APC, YWHAG, IKBKG, ARRB1, ESR1, FYN, GRB2, and NEDD4, ordered by their connection degrees decreasingly) are specifically noteworthy because they are the top 10 strongest-connected proteins in the processwise subnetwork (the union of the six clusters of protein interaction modules) and include the top 3 proteins with the highest betweenness as well (APC, YWHAZ, and IKBKG). Lowly-connected interfacing proteins are no less interesting, as they include the $\mathrm{HCV}$-binding protein SMURF2 and the cancer-mutated gene TPR, both of which have a connectivity of three. In all, many of these interfacing proteins are potentially related to $\mathrm{HCC}$, as 17 are covered in the HCC-responsive gene sets and five are targeted by $\mathrm{HCV}$ proteins (Additional file 1).

Of all 27 interfacing proteins, YWHAZ is the only one that interfaces with all six clusters of protein interaction modules (Figure 4). YWHAZ is a HCV-binding protein and is covered in two HCC-responsive gene sets (Additional file 1). Additionally, it has been implicated in HCC in terms of copy number alteration [35] and drug- responsive differential expression [36,37]. We extracted all protein interaction pairs (edges) connected to YWHAZ in the process-wise subnetwork and separated them into the six characteristic clusters (Figure 4). This YWHAZcenteredmodule contains four cancer-mutated genes, three HCV-binding proteins, and numerous HCCresponsive differential expression genes (Figure 4). Interestingly, three cancer-mutated genes had a similar differential co-expression pattern with YWHAZ, and all have a sharp correlation collapse in cirrhosis (Figure 4, cluster I). Although further investigation is warranted, the dynamic dissection of YWHAZ's interaction partners in this work provide unique clues to subtle mechanisms of HCC pathophysiology.

\section{Verification of the approach in an independent hepatocellular carcinoma dataset}

An independent gene expression dataset, GSE14323, was used to verify the N-C subnetwork. We observed that overall there was no correlation between the $d C$ values of the 56,142 overlapping protein interaction pairs in GSE6467 and GSE14323 $(r<0.01)$. Notably, the $d C$ values of the top $0.1 \%$ DCPs in GSE6467 (seed DCPs) are significantly positively correlated with those in GSE14323 $(r=0.23, p=$ 0.04 ). A similar significant positive correlation was observed for the $286 \mathrm{~N}-\mathrm{C}$ subnetwork protein interaction pairs between GSE6467 and GSE14323 $(r=0.10, p=0.04)$.

We then performed an analogous subnetwork search using GSE14323. Starting from top 1\% DCPs, we obtained

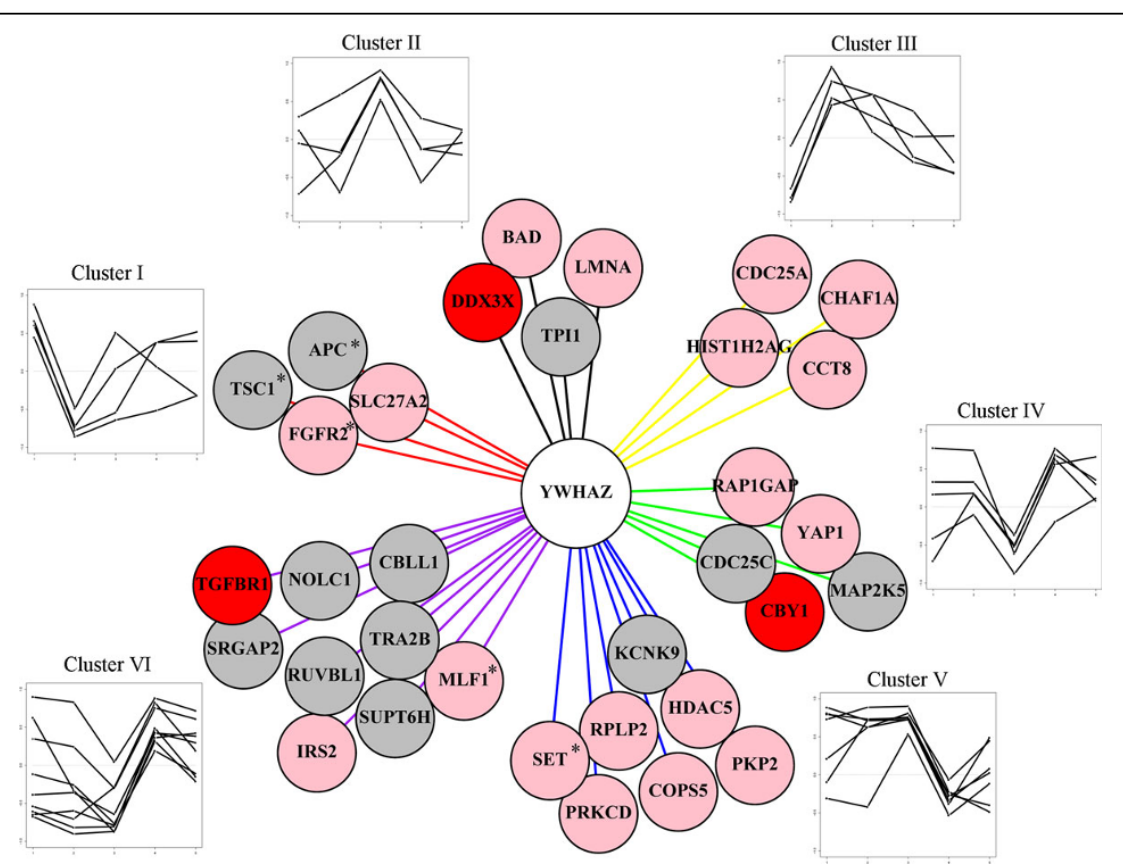

Figure 4 Thirty-three interactions of YWHAZ were categorized into six clusters based on their dynamic co-transcription profiles Hepatitis C virus protein-binding genes (in red), hepatocellular carcinoma-responsive genes (in pink), and cancer-mutated genes $\left({ }^{*}\right)$ were marked. 
a subnetwork with 255 links formed by 280 unique genes. There are 34 genes shared by the two N-C subnetworks, including one common hub - APC. FYN, a hub discovered in GSE14323 N-C subnetwork, is also a hub in E-A subnetwork of GSE6467. The GSE14323 subnetwork harbors an additional hub, CTNNB1, which is a confirmed HCCmutated gene [30]. Here, the recurrence of HCC-related hub genes from independent datasets indicates the validity of our differential co-expression network approach.

\section{Discussion}

In this work, we integrated differential co-expression analysis with protein interaction network and applied a network-based approach to uncover HCC-specific dynamic protein interaction modules. Our framework has generated a valuable set of plausibly HCC-implicated genes and protein interaction pairs for follow-up investigations. Currently, the number of assured HCC-implicated genes remains very small. In Cancer Gene Census [38], there are only five genes explicitly associated with liver cancer. Among these five genes, $A P C$ appears as a recurrent hub in our subnetworks (N-C and $\mathrm{C}-\mathrm{D}$ ), and it was also verified as a hub in the $\mathrm{N}-\mathrm{C}$ subnetwork using another independent dataset. In addition, some plausibly implicated genes stand out in our modules. These genes include those mutated in other cancer types and bound with HCV proteins (e.g. PIK3R1 and EP300), genes interfacing with alternative partner groups (e.g. YWHAZ), and genes manifesting both differential co-expression and differential expression (e.g. ESR1). Some genes meet multiple prioritization criteria; for instance, EEF1D is a DEG involved in a DCP and it interfaces with alternative partner groups. In summary, genes highlighted in our dynamic modules may serve as a set of practically plausible candidate targets for follow-up HCC studies.

Moreover, our framework provides a way to reveal protein interaction rewiring during HCC progression. For instance, alternative interaction partners were activated for the proteins shared in the N-C and C-D subnetworks. In our focused APC-centered module (Figure 2), two distinct groups of APC partners were distinguished by discriminating their correlation relationships with APC in the N-C-D progression: one group, consisting of CTNNA1 and NUPL1, demonstrated a NS-HN-NS (NS: non-significance; HN: High-Negative) correlation pattern with APC, while the other group, consisting of ZFP106, CYTH2, and HNRNPF, displayed an opposite NS-HP-NS (HP: High-Positive) pattern. Such alternative partnerships in company with condition changes could be actual instances of the "date hubs" conceptualized several years ago [39].

Comparing our differential co-expression subnetworks with previous differential expression subnetworks [24], we observed a significant number (21, hyper-geometric test, $p<0.001)$ of common genes if ignoring transitionto-transition mapping. This result indicates that a significant number of genes were remarkable in both differential co-expression and also differential expression, yet the two types of expression changes may not happen simultaneously. Interestingly, most of these shared genes manifested an earlier differential co-expression and then a later differential expression (Additional file 1). A similar trend was seen with the three genes overlapping the original differential expression study [25] (Additional file 1). This observation suggested that differential co-expression is a more upstream event than differential expression in biological systems. Along the central dogma, a causal mutation at the genetic level is unambiguously the most upstream event. Such a causal mutation is transduced through a conceptual biological information flow and ultimately results in consequences at the molecular, cellular, and bodily level. Closely succeeding the initial mutation event is transcription dysregulation, which sometimes manifests itself as "altered relationships" between regulators and targets or among targets. Differential expression, as the molecular-level output of the information flow, can occur with the causal regulator's direct targets as well as indirect targets. While a certain time-lag was necessary for a microRNA's regulatory effects to propagate fully to the secondary targets [40], it is likely that, at a higher-level and a larger scale, genes' differential expression phenomena may lag behind their differential co-expression circumstances by a time-phase that corresponds to one or more disease stages. This putative precedence of differential co-expression over differential expression deserves a systematic investigation in extended progressive disease datasets.

With the stage-wise HCC expression data, the proposed differential co-expression network approach resolved modules with coherent co-expression change patterns over all HCC developmental stages and even deciphered the temporal activation/dismissal of involved functions. In our results, negative regulation of the apoptotic process was found to be dismissed at early precancerous phases but was recruited in established HCC; positive regulation of the apoptotic process was to be coordinated in precancerous phase dysplasia. These mutually consistent functional dynamics may suggest broad, coordinated anti-cancer calibration mechanisms taking place in precancerous stages. Relating to the putative precedence of differential co-expression over differential expression, these functional dynamics may underpin pivotal HCC stage transitions for which more elaborate studies are warranted towards a potential early phase HCC intervention.

From a methodological point of view, our approach has some similarity to a few previous studies $[18,20,41]$. Like us, all of these studies have integrated protein 
interaction network with expression data, and their outputs have included sort of protein interaction subnetworks/modules. The most striking difference between our approach and the cited ones is that we first quantified differential co-expression of each protein interaction pair (edge) and then set out to search for the organization of those differentially co-expressed edges. Lin et al. [18] and Chuang et al. [20] were similar to each other in that they first constructed a co-expression protein interaction network for each disease state and then compared the two obtained network in terms of topological features or functional annotations; $\mathrm{Gu}$ et al. [41] devised a unique clustering framework which has taken into account both protein interaction network topology and gene expression correlation. As was lately proposed [42], differential co-expression network in which edges were weighted or dichotomized by direct differential coexpression measures (such as $d C$ in the present study) have distinctive topological features compared to traditional co-expression networks, so co-expression network-based strategies could not directly transfer to differential co-expression network studies. The ad hoc clustering algorithm by $\mathrm{Gu}$ et al. [41] could possibly be adapted for exploration of differential co-expression network, and it is of interest to see continuous improvement of these algorithms and hopefully a comparative evaluation of these related approaches will come out soon.

\section{Conclusions}

In this study, by integrating the protein interaction data and gene differential co-expression information, we sought to identify dynamic protein interaction modules from a hepatocellular carcinoma stage-wise expression dataset. We established the validity of searching for subnetworks from seeds of differential co-expressed gene pairs in contrast to traditional differential expression genes. Moreover, by examining the differential coexpression patterns associated with single stage-transitions or whole progression process, we revealed dynamic rewiring of protein interaction pairs and temporal activation/dismissal of pivotal functions in human hepatocellular carcinoma progression. Our framework has generated a valuable set of plausibly implicated genes and protein interaction pairs for follow-up human hepatocellular carcinoma investigations.

\section{Materials and methods}

Gene expression profiles and protein interaction network The HCC gene expression dataset GSE6764 [25] was obtained from Gene Expression Omnibus (GEO) [43]. It contains 20,068 genes and 75 samples. Three samples from cirrhotic liver tissue of non-HCC patients were excluded; the remaining 72 samples were classified into five stages of HCC development: Normal (N), Cirrhosis $(\mathrm{C})$, Dysplasia (D), Early HCC (E), and Advanced HCC (A). The numbers of samples included in these stages were 10, 10, 17,18 , and 17 , respectively.

Protein interaction pairs were downloaded on September 14, 2012 from the Protein Interaction Network Analysis (PINA) [44], which collected 70,297 protein interaction pairs between 12,373 proteins. After matching them with dataset GSE6764, we had a protein interaction network of 64,865 pairs between 10,953 proteins.

Another independent HCC gene expression dataset (GSE14323) [45] was used to verify the N-C transition results produced from the primary dataset GSE6764. Nineteen normal and 41 cirrhotic tissue samples of this dataset were used.

\section{HCC-related gene sets}

We compiled three sets of HCC-related genes as gold standards to evaluate the relevance of our results. The HCV-protein-binding (HCB) proteins were downloaded from the Hepatitis C Virus Protein Interaction Database [46] on October 17, 2012. The Cancer Gene Consensus (CGC) set included the cancer-mutated genes last updated on March 15, 2012 [47]. The HCC-responsive set (HCR) was a compendium of DEGs reported in HCC studies, compiled by querying the gene set database MSigDB [48] with the keywords "hepatocellular AND carcinoma." Mapping to our protein interaction network, we were left with $393 \mathrm{HCB}, 385 \mathrm{CGC}$, and 4,791 HCR genes, respectively.

\section{Discovery of differential co-expression subnetworks in $\mathrm{HCC}$}

We calculated the Pearson correlation coefficient $(r)$ for each gene pair under each HCC stage. Then, the Pearson correlation coefficients were transformed using Fisher's transformation [49]. Fisher's transformation could achieve a soft thresholding of the original $r$ values so that the larger $r$ values were emphasized while the smaller ones were downplayed. The transformed correlation value $\left(R_{k}\right)$ was calculated as in equation 1 :

$$
R_{k}=0.5 * \ln \frac{\left(1+r_{k}\right)}{\left(1-r_{k}\right)}
$$

where $r_{k}$ was untransformed Pearson correlation coefficient values with $k$ being $1,2,3,4$, or 5 (corresponding to the five HCC stages). After the Fisher's transformation, the transformed correlation value of each subsequent stage was subtracted from the counterpart values in the preceding stage, and a differential correlation value $(d C)$ was obtained as calculated by equation 2 . Consequently, four $d C$ values corresponding to the four stage transitions were assigned to each gene pair. 


$$
d C(k)=R_{k+1}-R_{k}
$$

As in the initial work [8] and our previous work [24], we searched for dense modules in protein interaction network from a beginning set of seeds. The procedure started from each of several initial network modules formed by top ranked DCPs (seeds) and ended with a combination of the iteratively expanded modules. At each iterative step, the module was growing outwardly by absorbing a directly-connecting edge with the maximum absolute $d C$ value, and it was assessed for its module score - the average absolute $d C$ value. Most often, the module score would decrease with the subnetwork expansion. The iteration continued if and only if the decreasing rate of the module score was not greater than delta and ended as the iteration exceeded 100 times. The strategy of the decreasing module score rate was applied to this approach, as we observed that the module scores often decreased in the first few steps during the expansion procedure. By applying the decreasing rate of module score, the chances of getting stuck at the early iteration stages were diminished; fragmented, small sized modules comprising most of the starting seeds could most likely be avoided. The upper-limit of iteration cycles was set at 100 to control the size of the resulting network modules. This subnetwork-searching algorithm was named the "edgewise dense module searching" (eDMS). An R script for eDMS is available upon request.

As stated in the previous work [8], delta is decisive in determining the final modules in network search. We followed the procedure from our previous work [24] to analyze a spectrum of delta (from 0 to 0.1 with an increase interval of 0.01). We then selected the optimal delta based on the overall module score - the average module score of disconnected components after eDMS ends. As in the previous work, we removed the disconnected components with less than five comprising genes before we reported the final subnetwork.

\section{Functional annotation for a set of genes}

GO [27] term enrichment analyses (in the "Biological Process" aspect) were performed using the hyper-geometric test provided in the R package GOstats [50], with the genes in the global protein interaction network taken as the universal background. For each GO term, the numbers of annotated genes from the background gene set and the foreground set (e.g. from a subnetwork or a set of hubs) were each identified. Then, a $p$-value indicative of the enrichment level of the GO term in question was calculated. After removing GO terms with four or less genes annotated from the foreground set, we adjusted the remaining GO terms' $p$-values using the Benjamini-Hochberg method [51]. GO terms with adjusted $p$-value larger than 0.001 (Clusters I, II, III, and V) or 0.01 (Cluster IV) were removed and only the most specific terms (leaf terms) of the remaining term set were reported.

\section{Additional material}

\begin{abstract}
Additional File 1: Supplementary results. This file contains all supplementary results that are not covered in the other additional files. Explanatory text, small tables, and small figures are included in this file.

Additional file 2: Seeds for subnetwork searches. This file documents the seed DCPS used for the four HCC-transition subnetwork searches.

Additional file 3: Transition-wise differential co-expression protein subnetworks. This file includes the visual display of all four transitionwise differential co-expression subnetworks.

Additional file 4: Edge statistics of transition-wise differential coexpression protein subnetworks. This file includes the statistics associated with all edges of the transition-wise differential co-expression subnetworks.

Additional file 5: Process-wise clusters of dynamic protein interaction modules. This file includes the visual display of the six clusters of process-wise differential co-expression protein interaction modules.

Additional file 6: Edge statistics of process-wise differential coexpression protein modules. This file includes the expression correlation values $(\boldsymbol{r})$ and differential co-expression values $(\boldsymbol{d} \boldsymbol{C})$ associated with edges from the six clusters of process-wise protein modules.
\end{abstract}

List of abbreviations used

CGC: Cancer Gene Consensus

GO: Gene Ontology

DEG: differentially expressed gene

DCG: differentially co-expressed gene

DCP: differentially co-expressed gene pair

HCB: HCV-protein-binding

HCC: Hepatocellular carcinoma

HCR: HCC-responsive

HCV: Hepatitis C virus

\section{Competing interests}

The authors declare that they have no competing interests.

\section{Authors' contributions}

$Z Z, H Y$, and $Y Y L$ conceived of the study and collected the data. HY performed the computational coding and implementation. $\mathrm{HY}$ and $\mathrm{CCL}$ conducted data analysis. $\mathrm{HY}, \mathrm{CCL}$, and $\mathrm{ZZ}$ drafted the manuscript. All authors read and approved the final manuscript.

\section{Acknowledgements}

The authors would like to thank Drs. Siyuan Zheng and Peilin Jia for insightful discussion and technical assistance, and Rebecca H. Posey for proofreading and editing an earlier draft of the manuscript. This work was partially supported by National Institutes of Health grants (R01LM011177, R03CA167695, P30CA68485, and P50CA095103), The Robert J. Kleberg, Jr. and Helen C. Kleberg Foundation (to Z.Z.), Ingram Professorship Funds (to Z. Z.), and grants from National Science Foundation of China (31171268, 31000380, and 81272279). The funders had no role in study design, data collection and analysis, decision to publish, or preparation of the manuscript.

\section{Declarations}

The publication costs for this article were funded by the corresponding author.

This article has been published as part of BMC Systems Biology Volume 7 Supplement 5, 2013: Selected articles from the International Conference on Intelligent Biology and Medicine (ICIBM 2013): Systems Biology. The full contents of the supplement are available online at http://www. biomedcentral.com/bmcsystbiol/supplements/7/S5. 


\section{Authors' details}

'Department of Biomedical Informatics, Vanderbilt University School of Medicine, Nashville, TN 37203, USA. ${ }^{2}$ Shanghai Center for Bioinformation Technology, Shanghai 201203, P. R. China. ${ }^{3}$ Department of Psychiatry, Vanderbilt University School of Medicine, Nashville, TN 37212, USA. ${ }^{4}$ Department of Cancer Biology, Vanderbilt University School of Medicine, Nashville, TN 37232, USA. ${ }^{5}$ Center for Quantitative Sciences, Vanderbilt University School of Medicine, Nashville, TN 37232, USA.

Published: 9 December 2013

\section{References}

1. De Las Rivas J, Fontanillo C: Protein-protein interaction networks: unraveling the wiring of molecular machines within the cell. Brief Funct Genomics 2012, 11:489-496.

2. Wu J, Vallenius T, Ovaska K, Westermarck J, Makela TP, Hautaniemi S: Integrated network analysis platform for protein-protein interactions. Nat Methods 2009, 6:75-77.

3. Sun J, Zhao Z: Functional features, biological pathways, and protein interaction networks of addiction-related genes. Chem Biodivers 2010, 7:1153-1162.

4. Guo AY, Sun J, Jia P, Zhao Z: Network analysis of ethanol-related candidate genes. Chem Biodivers 2010, 7:1142-1152.

5. Przytycka TM, Singh M, Slonim DK: Toward the dynamic interactome: it's about time. Brief Bioinform 2010, 11:15-29.

6. Chen B, Fan W, Liu J, Wu FX: Identifying protein complexes and functional modules-from static PPI networks to dynamic PPI networks. Brief Bioinform 2013.

7. Ideker T, Ozier O, Schwikowski B, Siegel AF: Discovering regulatory and signalling circuits in molecular interaction networks. Bioinformatics 2002, 18(Suppl 1):S233-240.

8. Chuang HY, Lee E, Liu YT, Lee D, Ideker T: Network-based classification of breast cancer metastasis. Mol Syst Biol 2007, 3:140.

9. Dittrich MT, Klau GW, Rosenwald A, Dandekar T, Muller T: Identifying functional modules in protein-protein interaction networks: an integrated exact approach. Bioinformatics 2008, 24:i223-231.

10. Sohler F, Hanisch D, Zimmer R: New methods for joint analysis of biological networks and expression data. Bioinformatics 2004, 20:1517-1521.

11. Nacu S, Critchley-Thorne R, Lee P, Holmes S: Gene expression network analysis and applications to immunology. Bioinformatics 2007, 23:850-858.

12. Camargo A, Azuaje F: Linking gene expression and functional network data in human heart failure. PLoS One 2007, 2:e1347.

13. Taylor IW, Linding R, Warde-Farley D, Liu Y, Pesquita C, Faria D, Bull S, Pawson T, Morris $\mathrm{Q}$, Wrana JL: Dynamic modularity in protein interaction networks predicts breast cancer outcome. Nat Biotechnol 2009, 27:199-204.

14. Guo Z, Wang L, Li Y, Gong X, Yao C, Ma W, Wang D, Zhu J, Zhang M, Yang $D$, et al: Edge-based scoring and searching method for identifying condition-responsive protein-protein interaction sub-network. Bioinformatics 2007, 23:2121-2128.

15. Xue H, Xian B, Dong D, Xia K, Zhu S, Zhang Z, Hou L, Zhang Q, Zhang Y, Han JD: A modular network model of aging. Mol Syst Biol 2007, 3:147.

16. Xiao Y, Xu C, Xu L, Guan J, Ping Y, Fan H, Li Y, Zhao H, Li X: Systematic identification of common functional modules related to heart failure with different etiologies. Gene 2012, 499:332-338.

17. Zhang $X$, Yang $H$, Gong B, Jiang C, Yang L: Combined gene expression and protein interaction analysis of dynamic modularity in glioma prognosis. J Neurooncol 2012, 107:281-288.

18. Lin CC, Hsiang JT, Wu CY, Oyang YJ, Juan HF, Huang HC: Dynamic functional modules in co-expressed protein interaction networks of dilated cardiomyopathy. BMC Syst Biol 2010, 4:138.

19. Yoon D, Kim H, Suh-Kim H, Park RW, Lee K: Differentially co-expressed interacting protein pairs discriminate samples under distinct stages of HIV type 1 infection. BMC Syst Biol 2011, 5(Suppl 2):S1.

20. Chung FH, Lee HH, Lee HC: ToP: A Trend-of-Disease-Progression Procedure Works Well for Identifying Cancer Genes from Multi-State Cohort Gene Expression Data for Human Colorectal Cancer. PLoS One 2013, 8:e65683.

21. de la Fuente A: From 'differential expression' to 'differential networking' identification of dysfunctional regulatory networks in diseases. Trends Genet 2010, 26:326-333.
22. Choi Y, Kendziorski C: Statistical methods for gene set co-expression analysis. Bioinformatics 2009, 25:2780-2786.

23. Yu H, Liu BH, Ye ZQ, Li C, Li YX, Li YY: Link-based quantitative methods to identify differentially coexpressed genes and gene pairs. $B M C$ Bioinformatics 2011, 12:315.

24. Zheng S, Tansey WP, Hiebert SW, Zhao Z: Integrative network analysis identifies key genes and pathways in the progression of hepatitis $C$ virus induced hepatocellular carcinoma. BMC Med Genomics 2011, 4:62.

25. Wurmbach E, Chen YB, Khitrov G, Zhang W, Roayaie S, Schwartz M, Fiel I, Thung S, Mazzaferro V, Bruix J, et al: Genome-wide molecular profiles of HCV-induced dysplasia and hepatocellular carcinoma. Hepatology 2007, 45:938-947.

26. Scott MS, Perkins T, Bunnell S, Pepin F, Thomas DY, Hallett M: Identifying regulatory subnetworks for a set of genes. Mol Cell Proteomics 2005, 4:683-692.

27. Ashburner M, Ball CA, Blake JA, Botstein D, Butler H, Cherry JM, Davis AP, Dolinski K, Dwight SS, Eppig JT, et al: Gene ontology: tool for the unification of biology. The Gene Ontology Consortium. Nat Genet 2000, 25:25-29.

28. Rehermann B, Nascimbeni M: Immunology of hepatitis B virus and hepatitis C virus infection. Nat Rev Immunol 2005, 5:215-229.

29. Sitia G, Aiolfi R, Di Lucia P, Mainetti M, Fiocchi A, Mingozzi F, Esposito A, Ruggeri ZM, Chisari FV, lannacone M, Guidotti LG: Antiplatelet therapy prevents hepatocellular carcinoma and improves survival in a mouse model of chronic hepatitis B. Proc Natl Acad Sci USA 2012, 109:E2165-2172.

30. Guichard C, Amaddeo G, Imbeaud S, Ladeiro Y, Pelletier L, Maad IB, Calderaro J, Bioulac-Sage P, Letexier M, Degos F, et al: Integrated analysis of somatic mutations and focal copy-number changes identifies key genes and pathways in hepatocellular carcinoma. Nat Genet 2012, 44:694-698.

31. Pathway Interaction Database. [http://pid.nci.nih.gov/]

32. Sygut A, Przybylowska K, Ferenc T, Dziki L, Spychalski M, Mik M, Dziki A: Genetic Variations of the CTNNA1 And The CTNNB1 Genes in Sporadic Colorectal Cancer in Polish Population. Pol Przegl Chir 2012, 84:560-564.

33. Ramakrishna G, Anwar T, Angara RK, Chatterjee N, Kiran S, Singh S: Role of cellular senescence in hepatic wound healing and carcinogenesis. Eur J Cell Biol 2012, 91:739-747.

34. Calvisi DF, Ladu S, Gorden A, Farina M, Conner EA, Lee JS, Factor VM, Thorgeirsson SS: Ubiquitous activation of Ras and Jak/Stat pathways in human HCC. Gastroenterology 2006, 130:1117-1128.

35. Jia D, Wei L, Guo W, Zha R, Bao M, Chen Z, Zhao Y, Ge C, Zhao F, Chen T, et al: Genome-wide copy number analyses identified novel cancer genes in hepatocellular carcinoma. Hepatology 2011, 54:1227-1236.

36. Wang J, Chan JY, Fong CC, Tzang CH, Fung KP, Yang M: Transcriptional analysis of doxorubicin-induced cytotoxicity and resistance in human hepatocellular carcinoma cell lines. Liver Int 2009, 29:1338-1347.

37. Ye F, Che Y, McMillen E, Gorski J, Brodman D, Saw D, Jiang B, Zhang DY: The effect of Scutellaria baicalensis on the signaling network in hepatocellular carcinoma cells. Nutr Cancer 2009, 61:530-537.

38. Futreal PA, Coin L, Marshall M, Down T, Hubbard T, Wooster R, Rahman N, Stratton MR: A census of human cancer genes. Nat Rev Cancer 2004, 4:177-183.

39. Han JD, Bertin N, Hao T, Goldberg DS, Berriz GF, Zhang LV, Dupuy D, Walhout AJ, Cusick ME, Roth FP, Vidal M: Evidence for dynamically organized modularity in the yeast protein-protein interaction network. Nature 2004, 430:88-93.

40. Tu K, Yu H, Hua YJ, Li YY, Liu L, Xie L, Li YX: Combinatorial network of primary and secondary microRNA-driven regulatory mechanisms. Nucleic Acids Res 2009, 37:5969-5980

41. Gu J, Chen Y, Li S, Li Y: Identification of responsive gene modules by network-based gene clustering and extending: application to inflammation and angiogenesis. BMC Syst Biol 2010, 4:47.

42. Bhattacharyya M, Bandyopadhyay S: Studying the differential coexpression of microRNAs reveals significant role of white matter in early Alzheimer's progression. Mol Biosyst 2013, 9:457-466.

43. Gene Expression Omnibus. [http://www.ncbi.n/m.nih.gov/geo/]

44. Cowley MJ, Pinese M, Kassahn KS, Waddell N, Pearson JV, Grimmond SM, Biankin AV, Hautaniemi S, Wu J: PINA v2.0: mining interactome modules. Nucleic Acids Res 2012, 40:D862-865

45. Mas VR, Maluf DG, Archer K, Yanek K, Kong X, Kulik L, Freise CE, Olthoff KM, Ghobrial RM, Mclver P, Fisher R: Genes involved in viral carcinogenesis 
and tumor initiation in hepatitis $C$ virus-induced hepatocellular carcinoma. Mol Med 2009, 15:85-94.

46. Hepatitis C Virus Protein Interaction Database. [http://cbrc.kaust.edu.sa/ hevpro/].

47. Cancer Gene Consensus. [http://www.sanger.ac.uk/genetics/CGP/Census/ symbol.shtml].

48. MSigDB. [http://www.broadinstitute.org/gsea/msigdb/search.jsp].

49. Fisher RA: Frequency Distribution of the Values of the Correlation Coefficient in Samples from an Indefinitely Large Population. Biometrika 1915, 10:507-521.

50. Falcon $\mathrm{S}$, Gentleman R: Using GOstats to test gene lists for GO term association. Bioinformatics 2007, 23:257-258.

51. Benjamini $Y, Y$. H: Controlling the false discovery rate: a practical and powerful approach to multiple testing. J R Statist SoC B 1995, 57:289-300.

doi:10.1186/1752-0509-7-S5-S2

Cite this article as: Yu et al.: Dynamic protein interaction modules in human hepatocellular carcinoma progression. BMC Systems Biology 2013 7(Suppl 5):S2.

\section{Submit your next manuscript to BioMed Central} and take full advantage of:

- Convenient online submission

- Thorough peer review

- No space constraints or color figure charges

- Immediate publication on acceptance

- Inclusion in PubMed, CAS, Scopus and Google Scholar

- Research which is freely available for redistribution

Submit your manuscript at www.biomedcentral.com/submit 